\title{
Knockdown of clusterin inhibits the growth and migration of renal carcinoma cells and leads to differential gene expression
}

\author{
HUA SHI $^{1}$, JUN-HONG DENG $^{1}$, ZHU WANG $^{2}$, KAI-YUAN CAO $^{2}$, LIANG ZHOU $^{1}$ and HUA WAN ${ }^{1}$ \\ ${ }^{1}$ Department of Urology, The First People's Hospital Affiliated to Guangzhou Medical University, \\ Guangzhou 510180; ${ }^{2}$ Research Center for Clinical Laboratory Standard, Department of Medical Microbiology, \\ Zhongshan Medical School of Sun Yat-sen University, Guangzhou 510080, P.R. China
}

Received December 19, 2012; Accepted April 11, 2013

DOI: $10.3892 / \mathrm{mmr} .2013 .1470$

\begin{abstract}
Clusterin (CLU) is a glycoprotein involved in tumor progression, whose expression level correlates with the metastasis of renal cell carcinoma (RCC). However, the mechanism by which CLU plays an oncogenic role in RCC remains unclear. In this study, we used the human renal cancer cell 786-O as an experimental model. We knocked down CLU expression in the 786-O cells using lentiviral vector-mediated delivery of RNAi, and then compared the gene expression profiles between the knocked down CLU 786-O cells and control cells. We observed that CLU knockdown induced apoptosis and inhibited the proliferation and migration of $786-O$ cells. Microassay analysis revealed changes in the expression of 588 genes between the 786-O cells infected by a si-CLU lentivirus and the control cells, where 356 genes were upregulated and 232 were downregulated. Pathway analysis classified the differentially expressed genes into 17 upregulated and 12 downregulated pathways, including the PI3K/Akt, MAPK and VEGF pathways. In this study, we demonstrated that CLU acts as an oncogene in RCC by promoting cell proliferation and migration and inhibiting apoptosis. Microassay analysis may provide a platform for further characterization of the individual genes implicated in the development of RCC, providing new insights into the oncogenic role of CLU.
\end{abstract}

\section{Introduction}

Renal cell carcinoma (RCC) is the most common primary renal malignant neoplasm in adults. It accounts for $~ 3 \%$ of adult malignancies and $90-95 \%$ of renal neoplasms. The gold standard for RCC treatment is surgery, where nephron-sparing surgery, laparoscopic and robotic surgery and minimally invasive procedures have all decreased the morbidity of RCC (1).

Correspondence to: Professor Jun-Hong Deng, Department of Urology, The First People's Hospital Affiliated to Guangzhou Medical University, No.1 Panfu Road. Guangzhou 510180, P.R. China E-mail: drdjhgz@163.com

Key words: RNA interference, clusterin, renal cell carcinoma, 786-O cells, microarray
However, advanced or metastatic RCC may develop resistance to chemotherapy or radiotherapy, contributing to a poor prognosis (2). In order to develop effective therapeutic strategies for RCC, further investigations are required to understand the molecular pathogenesis of aggressive RCC.

Clusterin (CLU), also known as testosterone repressed prostate message-2 or sulfated glycoprotein-2, is a glycoprotein crucial to various pathophysiological processes (3), such as tumor pathogenesis and progression. CLU is overexpressed in a variety of tumors, including in liver, pancreatic, colorectal, ovarian, prostate, bladder and kidney cancer (4). Furthermore, CLU expression levels correlate with the metastasis of melanoma, gastric cancer, ovarian cancer and RCC (5-8). However, the molecular mechanism by which CLU plays an oncogenic role in $\mathrm{RCC}$ remains unclear.

Global expression analysis using microarrays may be able to monitor the expression of thousands of genes in a high-throughput manner to provide novel insights into the mechanisms of cancer initiation, progression, resistance to treatment and response to cellular microenvironments (9). Therefore, in the present study, we used the human renal cancer cell line 786-O as an experimental model. We knocked down CLU expression in 786-O cells using lentiviral vector-mediated delivery of RNAi, and then compared the gene expression profiles of knocked down CLU 786-O cells and control cells. We demonstrated that CLU knockdown induces apoptosis and inhibits the proliferation and migration of 786-O cells. Furthermore, we identified differentially expressed genes after CLU knockdown and analyzed the related pathways in which these genes are involved.

\section{Materials and methods}

Cell culture. The 786-O cell line was purchased from the American Type Culture Collection (ATCC, Manassas, VA, USA) and cultured in RPMI-1640 medium (HyClone, Logan, UT, USA) supplemented with $10 \% \mathrm{Gibco}^{\mathrm{TM}} \mathrm{FBS}$ (Life Technologies, Grand Island, NY, USA) at $37^{\circ} \mathrm{C}$ in a standard humidified incubator containing $5 \% \mathrm{CO}_{2}$ and $95 \% \mathrm{O}_{2}$. The study was approved by the Ethics Committee of The First People's Hospital Affiliated to Guangzhou Medical University (Guangzhou, China). 
Lentivirus RNAi construct and transfection. The following three siRNA sequences targeting human CLU were provided by GeneChem Co., Ltd. (Shanghai, China): 1, 5'-CAGGGAAG TAAGTACGTCAATCTCGAGATTGACGTACTTACTTCCC TGTTTTT-3'; 2, 5'-GCTAAAGTCCTACCAGTGGAACTCG AGTTCCACTGGTAGGACTTTAGCTTTTT-3'; and 3, 5'-AGGGAAGTAAGTACGTCAATACTCGAGTATTGAC GTACTTACTTCCCTTTTTT-3'. A control siRNA with non-specific sequences was also produced. These siRNAs were cloned into pGCSIL-GFP plasmids. Lentiviruses were generated by the transfection of $80 \%$ confluent HEK293T cells with recombinant pGCSIL-GFP plasmids and pHelper 1.0 and pHelper 2.0 helper plasmids (GeneChem Co., Ltd.) using Lipofectamine 2000 (Invitrogen, Carlsbad, CA, USA). Lentiviruses were harvested in serum-free medium after 2 days, filtered and concentrated in primed Centricon Plus-20 filter devices (Millipore, Billerica, MA, USA), and the titers of recombinant lentiviruses were determined.

The 786-O cells were seeded in 6-well plates and grown to $60 \%$ confluence on the day of transfection. Four hours prior to transfection, cells were placed in serum-free media. The cells were transfected with a titrated siRNA vector diluted in RPMI-1640, with the addition of $1 \mu$ l polybrene. Successful knockdown of CLU was analyzed by real-time PCR and western blot analysis.

Real-time PCR assay. Total RNA was extracted from the $786-\mathrm{O}$ cells using TRIzol (Invitrogen), according to the manufacturer's instructions. First-strand cDNA was generated from $2 \mu \mathrm{g}$ total RNA using the PrimeScript RT reagent kit (Takara, Dalian, China) with random primers. Real-time PCR was performed on an ABI Prism 7300 (Applied Biosystems, Foster City, CA, USA). The specific primers were as follows: CLU, 5'-TCCGCGGCAT TCTTTGGGCG-3' and 5'-GCACTGGGAGGCGCCGTATT-3'; and $\beta$-actin, 5'-CGGAGTCAACGGATTTGGTCGTAT-3' and 5'-CCTTGCACATGCCGGAGCCGT-3'. Thermal cycling was initiated with a denaturation step for $1 \mathrm{~min}$ at $95^{\circ} \mathrm{C}$ followed by 40 cycles performed in two steps; $5 \mathrm{sec}$ at $95^{\circ} \mathrm{C}$ and $30 \mathrm{sec}$ at $60^{\circ} \mathrm{C}$. The relative mRNA level of CLU was compared to that of $\beta$-actin and was calculated by the $2^{-\Delta \Delta \mathrm{Ct}}$ method. Each $\mathrm{Ct}$ value used for these calculations was the mean of triplicate results obtained for each reaction.

Western blot analysis. The 786-O cells were lysed in RIPA buffer supplemented with protease inhibitors. The supernatant was collected for the protein concentration assay. Equal amounts of protein $(30 \mu \mathrm{g})$ were separated in $10 \%$ SDS-polyacrylamide gels and transferred to PVDF membranes. The membranes were blocked using non-fat milk in TBST and probed with either a CLU (Abcam, Cambridge, MA, USA) or GAPDH antibody (Santa Cruz Biotech, Santa Cruz, CA, USA), followed by incubation with a secondary antibody. Immunoreactivity signals were developed using an enhanced chemiluminescence (ECL) kit (GE Healthcare, Piscataway, NJ, USA).

Cell proliferation assay. The proliferation of 786-O cells was assessed using a WST-1 kit (Beyotime, Haimen, China), according to the manufacturer's instructions. After lentivirus infection, the cells were seeded in 6-well plates and incubated at $37^{\circ} \mathrm{C}$. Cell proliferation was assessed based on the absorbance measured at $450 \mathrm{~nm}$ using a multiwell spectrophotometer (Eppendorff, Hamburg, Germany).

Wound healing assay. After lentivirus infection, 786-O cells were seeded at $5 \times 10^{5}$ cells/well in 6 -well plates and cultured in RPMI-1640 medium supplemented with 10\% FBS for $\sim 24 \mathrm{~h}$ to near confluence. The cell monolayer was scraped in a straight line using a $20 \mu \mathrm{l}$ pipette tip to create a scratch, and the medium was changed to remove detached cells. Images were captured at $0,6,12$ and $24 \mathrm{~h}$ after scratching and analyzed using the Image $\mathrm{J}$ program to calculate cell migration distance. Cells from six representative fields were counted.

Flow cytometry analysis of apoptosis. Apoptosis was evaluated using annexin V/propidium iodide (PI; BD-Biosciences, San Jose, CA, USA) staining followed by flow cytometry analysis. After lentivirus infection, the cells were plated in 6 -well plates at a density of $1 \times 10^{6}$ cells/well, cultured at $37^{\circ} \mathrm{C}$ in a $5 \% \mathrm{CO}_{2}$ incubator for three days, then gently trypsinized and washed with ice-cold PBS. The cells were resuspended in $500 \mu 11 \mathrm{X}$ binding buffer and stained with annexin V and PI. The samples were subjected to flow cytometry analysis within $1 \mathrm{~h}$ using a flow cytometer (BD LSRII; BD-Biosciences) and the data were analyzed using BD FACS Diva software (BD-Biosciences).

Microarray analysis. Total RNA was extracted from 786-O cells using TRIzol (Invitrogen), according to the manufacturer's instructions, and purified with an RNeasy Mini kit (Qiagen, Mississauga, Ontario, ON, Canada). The integrity of the purified RNA was examined using agarose gel electrophoresis, and the quality and quantity of purified RNA were assessed using an Agilent 2100 Bioanalyzer RNA 6000 NanoChip (Agilent, Palo Alto, CA, USA). Only RNA samples with an A260/A280 between 1.7 and 2.2 were used in further experiments. Total RNA (5 $\mu \mathrm{g})$ was used to generate cDNA, which was labeled with the NimbleGen one-color DNA labeling kit (Roche NimbleGen, Madison, WI, USA). The labeled cDNA was hybridized in a NimbleGen human gene expression 12x135 K microarray (Roche NimbleGen, Madison, WI, USA), according to the manufacturer's instructions. The washed arrays were spin-dried and scanned using the Genepix 4000B scanner (Axon Instruments, Union City, CA, USA).

Microarray data analysis. Images were extracted and processed using NimbleScan v2.4 software and analyzed using the NimbleGen software (both Roche NimbleGen). The lognormal-normal model was employed in order to estimate the expression of each gene. Genes were ranked according to this value and the results were filtered further according to the magnitude of the change in expression; only genes that were at least 2-fold upregulated or downregulated were considered. Gene Ontology (GO) analysis for the differentially expressed genes was performed by using DAVID (http://david.abcc. ncifcrf.gov/. Accessed April 18, 2013), and Kyoto Encyclopedia of Genes and Genomes (KEGG) pathways were analyzed using the Ingenuity Pathway Analysis software package (IPA; Ingenuity Systems, Redwood City, CA, USA). 


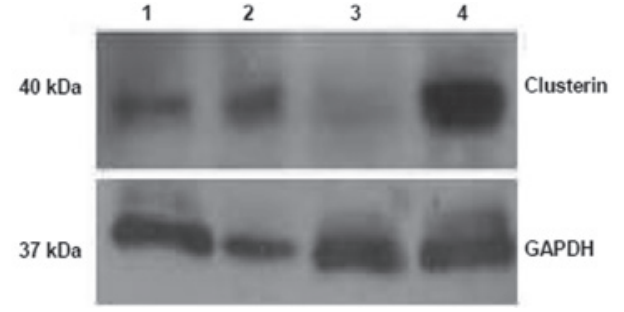

Figure 1. Knockdown of clusterin (CLU) expression in 786-O cells. Western blots demonstrating CLU protein levels in 786-O cells infected with different lentiviruses. GAPDH was used as a loading control.Lanes 1, CLU-RNAi-LV1; 2, CLU-RNAi-LV2; 3, CLU-RNAi-LV3; and 4, control siRNA.
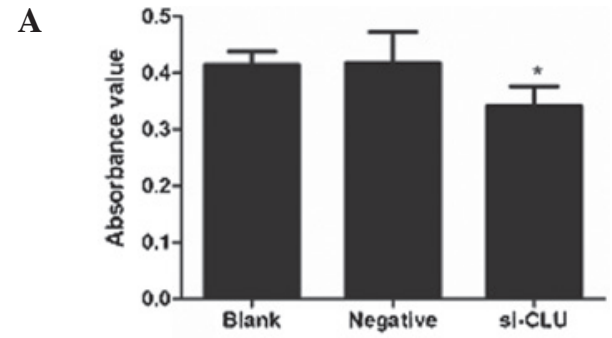

B

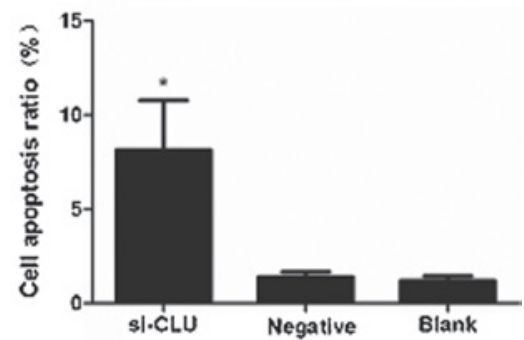

Figure 2. CLU knockdown inhibits the proliferation and induces the apoptosis of 786-O cells. (A) WST-1 assay showing the absorbance value in different groups of 786-O cells. CLU knockdown significantly reduced the proliferation of 786-O cells. (B) Flow cytometry assay showing the ratios of apoptosis in different groups of 786-O cells. CLU knockdown induced apoptosis in the $786-O$ cells. Data were presented as the mean \pm SD, $n=3$. $P<0.05$ vs. the blank and negative control groups. Blank, uninfected cells; negative, infected by control siRNA lentivirus; si-CLU, infected by CLU siRNA lentivirus.

Statistical analysis. Values were represented as the means \pm SD for at least triplicate determination, and analyzed using one-way ANOVA and an LSD test. All statistical analyses were performed using SPSS 13.0 , where $\mathrm{P}<0.05$ was considered to indicate a statistically significant difference.

\section{Results}

Evaluation of $C L U$ knockdown in 786-O cells. To verify that the CLU-RNAi lentivirus efficiently knocked down CLU in 786-O cells, we performed real-time PCR analysis to detect CLU mRNA levels in 786-O cells transduced by a different CLU-RNAi lentivirus and a negative control lentivirus. The results demonstrated that no. 3 CLU RNAi was most the efficient at reducing the CLU mRNA levels (data not shown). We performed western blot analysis to detect the CLU protein levels in the 786-O cells transduced by a different lentivirus. The results demonstrated that no. 3 CLU RNAi was the most efficient at reducing the CLU protein levels (Fig. 1), consistent with real-time PCR results. Therefore, we chose

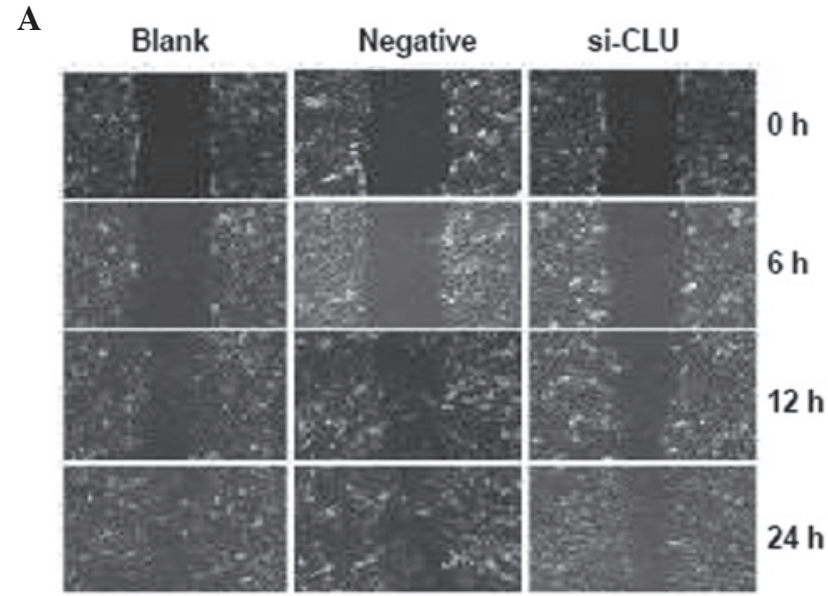

B

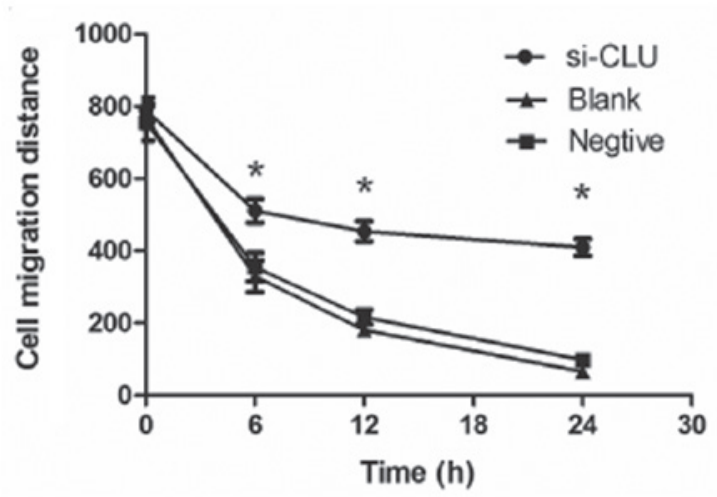

Figure 3. Clusterin (CLU) knockdown inhibits the migration of 786-O cells. (A) Representative images showing the migration of 786-O cells at $0,6,12$ and $24 \mathrm{~h}$ after scratches were made in the cell monolayer (magnification, $\mathrm{x} 200$ ). (B) Statistical analysis of cell migration distance in the wound healing assay at the time points shown in (A). CLU knockdown reduced the migration of 786-O cells. Data were presented as the mean $\pm S D, n=3$. ${ }^{*} P<0.05$ vs. the blank and negative control groups. Blank, uninfected cells; negative, infected by control siRNA lentivirus; si-CLU, infected by CLU siRNA lentivirus.

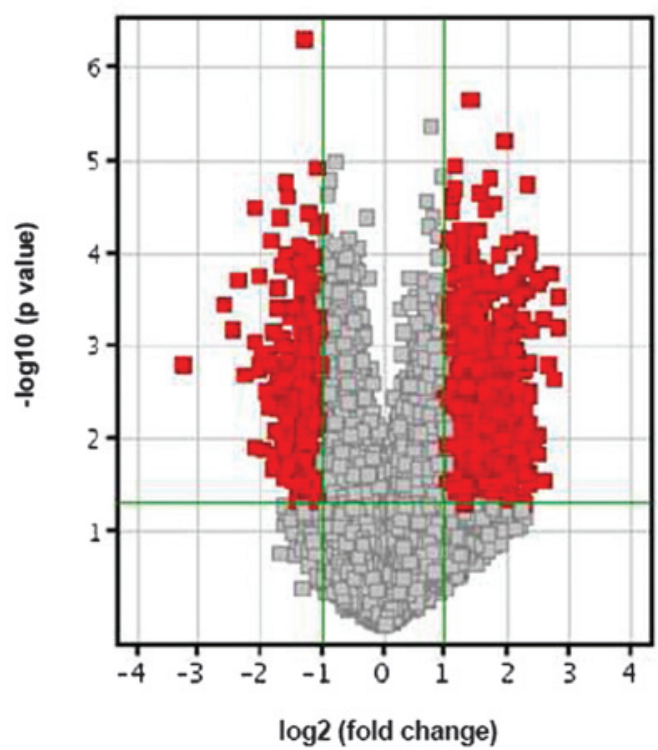

Figure 4. Genes differentially expressed in the 786-O cells after clusterin (CLU) knockdown. The volcano plot demonstrates the differential expression of the illustrated genes; dots in grey represent genes that did not achieve significant changes in expression, dots in red on the left indicate the genes with significantly downregulated expression and dots in red on the right indicate the genes with significantly upregulated expression. 
Table I. Biological pathways of differentially expressed genes in clusterin (CLU) knockdown 786-O cells.

$\begin{array}{ll}\text { Pathways } & \text { Genes }\end{array}$

Upregulated

Viral myocarditis

Hematopoietic cell lineage

Graft-versus-host disease

Basal cell carcinoma

Type 1 diabetes mellitus

Leishmaniasis

Staphylococcus aureus infection

Hedgehog signaling

Autoimmune thyroid disease

Allograft rejection

Phagosome

Antigen processing and presentation

Intestinal immune network for Ig A

ECM-receptor interaction

Cytokine-receptor interaction

Toxoplasmosis

HTLV-I infection

Downregulated

Focal adhesion

Carbohydrate digestion and absorption

Small cell lung cancer

Glioma

Prostate cancer

mTOR signaling pathway

MAPK signaling pathway

\section{Melanoma}

Non-small cell lung cancer

Chronic myeloid leukemia

Aldosterone regulated sodium reabsorption

Pathways in cancer
HLA-A, HLA-DPB 1, HLA-DQB 1, HLA-DRB 1, HLA-DRB1, HLA-DRB3, HLA-G, ITGB2, MYH13

CD7, GP1BA, GP9, HLA-DRB1, HLA-DRB1, HLA-DRB3, IL5RA, IL9R, ITGAM, TFRC

HLA-A, HLA-DPB 1, HLA-DQB1, HLA-DRB1, HLA-DRB1, HLA-DRB3, HLA-G

DVL1, PTCH2, WNT1, WNT2, WNT3, WNT3A

HLA-A, HLA-DPB 1, HLA-DQB1, HLA-DRB 1, HLA-DRB1, HLA-DRB3, HLA-G

FCGR2C, HLA-DPB1, HLA-DQB1, HLA-DRB1, HLA-DRB1, HLA-DRB3, ITGAM, ITGB2, NCF1

FCGR2C, FPRL2, HLA-DPB1, HLA-DQB1, HLA-DRB1, HLA-DRB1, HLA-DRB3, ITGAM, ITGB2

BMP8B, PRKACG, PTCH2, WNT1, WNT2, WNT3, WNT3A

HLA-A, HLA-DPB1, HLA-DQB1, HLA-DRB1, HLA-DRB1, HLA-DRB3, HLA-G, IFNA4

HLA-A, HLA-DPB 1, HLA-DQB1, HLA-DRB 1, HLA-DRB1, HLA-DRB3, HLA-G

CLEC4M, COMP, FCGR2C, HLA-A, HLA-DPB1, HLA-DQB1,

HLA-DRB1,HLA-DRB1, HLA-DRB3, HLA-G, ITGAM, ITGB2, NCF1, TFRC, TUBB2B

HLA-A, HLA-DPB 1, HLA-DQB1, HLA-DRB 1, HLA-DRB1, HLA-DRB3, HLA-G, HSPA6, KIR2DL4

CCL25, HLA-DPB1, HLA-DQB1, HLA-DRB1, HLA-DRB1, HLA-DRB3, MADCAM1

COL11A2, COMP, GP1BA, GP9, ITGB4, LAMA5, LAMC3, SDC3

BLR1, CCL1, CCL25, CCL4L2, CCL4L2, CLC, EDA, IFNA4, IL17B, IL5RA, IL9R, PF4, TNFRSF25, TNFRSF6B, TNFSF14, XCL1

AKT1, BIRC4, HLA-DPB1, HLA-DQB1, HLA-DRB1, HLA-DRB1, HLA-DRB3, HSPA6, LAMA5, LAMC3, PLA2G2F

AKT1, ATM, BIRC4, DVL1, HLA-A, HLA-DPB1, HLA-DQB1, HLA-DRB1,HLA-DRB1, HLA-DRB3, HLA-G, ITGB2, PRKACG, WNT1, WNT2, WNT3,WNT3A

AKT3, ARHGAP5, EGFR, FN1, ITGA5, ITGB3, PARVA, PDGFC, PDPK1, PIK3CD, PRKCA, RAP1B, VEGF

AKT3, ATP1A1, ATP1A1, HK2, PIK3CD

AKT3, CDK6, FN1, PIAS2, PIK3CD, RB1, RXRA

AKT3, CDK6, EGFR, MDM2, PIK3CD, PRKCA, RB1

AKT3, CREB3L2, EGFR, MDM2, PDGFC, PDPK1, PIK3CD, RB1

AKT3, DDIT4, PDPK1, PIK3CD, RICTOR, RPS6KA2, VEGF

AKT3, ATF2, CASP3, DUSP5, EGFR, FGF5, IL1R1, MAP3K7IP2, MAP3K8, MAPK14, MAPKAPK2, NF1, PRKACB, PRKCA, RAP1B, RAPGEF2, RPS6KA2

AKT3, CDK6, EGFR, FGF5, MDM2, MITF, PDGFC, PIK3CD, RB1

AKT3, CDK6, EGFR, PDPK1, PIK3CD, PRKCA, RB1, RXRA

AKT3, CBLB, CDK6, MDM2, PIK3CD, RB1, RUNX1

ATP1A1, ATP1A1, PDPK1, PIK3CD, PRKCA

AKT3, AXIN2, CASP3, CBLB, CCDC6, CDK6, CUL2, EGFR, EGLN1, FGF5, FN1, GLI2, KITLG, MDM2, MITF, PIAS2, PIK3CD, PRKCA, RB1, RUNX1, RXRA, VEGF, WNT5A 
no. 3 CLU RNAi to knockdown CLU in 786-O cells in subsequent experiments.

CLU knockdown inhibits the proliferation of RCC cells. To investigate whether CLU regulates the proliferation of RCC cells, 786-O cells were infected with either an si-CLU lentivirus or a control lentivirus, and cell proliferation was evaluated with a WST-1 assay. The results revealed that CLU knockdown reduced the proliferation of 786-O cells over the $72 \mathrm{~h}$ period (Fig. 2A).

The effect of CLU knockdown on apoptosis in RCC cells. Flow cytometry analysis revealed that the apoptotic ratio was $6.30 \pm 3.17 \%$ in $786-O$ cells infected by the si-CLU lentivirus, which was significantly lower than that in cells infected by the negative control lentivirus $(1.20 \pm 0.40 \%)$ or uninfected cells (1.01 $\pm 0.37 \%$; Fig. 2B). These results suggest that CLU plays an anti-apoptotic role to promote the proliferation of RCC cells.

CLU knockdown inhibits the migration of RCC cells. To investigate whether CLU regulates the migration of RCC cells, an important behavior involved in RCC metastasis, 786-O cells were infected with either an si-CLU lentivirus or a control lentivirus, and cell migration was evaluated using a wound healing assay. We observed that CLU knockdown reduced the migration of 786-O cells at 12 and $24 \mathrm{~h}$ after scratches were created (Fig. 3), suggesting that CLU promotes the migration and invasion of RCC cells.

CLU knockdown leads to differential gene expression in RCC cells. To identify gene regulation networks that contribute to the various biological behaviors of 786-O cells upon CLU knockdown, we performed microarray analysis to compare the gene expression profiling in 786-O cells infected by si-CLU lentivirus vs. cells infected by a negative control lentivirus. Notably, 588 genes showed significant changes in expression between the 786-O cells infected with an si-CLU lentivirus and the control cells $(\mathrm{P}<0.01)$, with 356 genes upregulated and 232 downregulated (Fig. 4). These differentially expressed genes were distributed in almost all chromosomes, with the exception of the $\mathrm{Y}$ chromosome, but were enriched in chromosome 1 (9.97\%), chromosome 2 (7.58\%), chromosome $6(5.92 \%)$, chromosome $11(8.1 \%)$, chromosome $14(5.4 \%)$ and chromosome 19 (5.92\%).

We performed GO and KEGG pathway analyses to classify the differentially expressed genes in the 786-O cells after CLU knockdown. The results demonstrated that 17 pathways were upregulated and 12 were downregulated (Table I).

\section{Discussion}

CLU is overexpressed in a variety of tumors and promotes tumorigenesis. Furthermore, 3-CLU has been proposed as a prognostic marker for RCC (10). A recent study reported that an antisense oligodeoxynucleotide targeting clusterin exhibited antitumor activity in an RCC model (11). These studies suggest that CLU plays an oncogenic role in RCC. Consistent with this theory, in the present study, we employed a loss-of-function approach to knockdown CLU in the 786-O cell line and observed that CLU knockdown induces apoptosis and inhibits the proliferation and migration of 786-O cells. These results provide further evidence for the oncogenic role of CLU in RCC. However, the detailed molecular mechanisms by which CLU promotes RCC development remain largely unknown.

Cancer development is known to be a multi-step process involving sequential changes in a variety of genes and cellular pathways (12). Microarray techniques have been applied widely in cancer research due to their advantages in revealing the dynamics of gene expression and gene regulation networks from a global perspective, which contributes to our understanding of cancer initiation, progression and metastasis $(9,13,14)$.

In this study, we used a NimbleGen microarray to screen the differentially expressed genes in 786-O cells after CLU knockdown vs. the parental $786-\mathrm{O}$ cells. We revealed that 356 genes were upregulated and 232 were downregulated. Although these differentially expressed genes were distributed in almost all chromosomes, with the exception of the Y chromosome, they were relatively enriched in chromosomes 1 , 2, 6, 11 and 14, consistent with previous molecular genetics studies on RCC. For example, Beroukhim et al (15) identified 7 regions of deletion (1p, 3p, 4q, 6q, 8p, 9p and 14q) in hereditary and sporadic clear-cell RCC. Moreover, Monzon et al (16) identified deletions in chromosomes 1, 5, 6, 9, 13 and 14 in renal cancer patients.

Furthermore, we classified the differentially expressed genes into different biological pathways in order to characterize their functional role in RCC. As expected, approximately half of the downregulated pathways are cancer-related, including the PI3K/Akt, MAPK and VEGF pathways, known to promote cancer cell proliferation, survival and tumor angiogenesis and metastasis. After CLU knockdown, the downregulation of these pathways may have contributed to the observed inhibition of 786-O cell proliferation and migration. Notably, we identified that a number of pathways involved in immunity and infection were upregulated. Future studies exploring the correlation between RCC development and immunological function may shed new light on the functional role of CLU in tumorigenesis.

In conclusion, this study presents evidence that CLU acts as an oncogene in RCC by promoting cancer cell proliferation and migration and inhibiting cancer cell apoptosis. We identified differentially expressed genes after CLU knockdown and classified them according to their related biological pathways. Our findings provide a platform for further characterization of the individual genes implicated in RCC development, which may provide new insights into the oncogenic role of CLU.

\section{Acknowledgements}

This work was supported by the grants from the Science and Technology Fund of Guangdong Province (no. 2009B030801053) and the Science and Technology Fund of Guangzhou City (no. 2009Z1-E381-02).

\section{References}

1. Patel C, Ahmed A and Ellsworth P: Renal cell carcinoma: a reappraisal. Urol Nurs 32: 182-190, 2012.

2. Cohen HT and McGovern FJ: Renal cell carcinoma. N Engl J Med 353: 2477-2490, 2005. 
3. Rosenberg ME and Silkensen J: Clusterin: physiologic and pathophysiologic considerations. Int J Biochem Cell Biol 27: 633-645, 1995

4. Shannan B, Seifert M, Leskov K, et al: Challenge and promise: roles for clusterin in pathogenesis, progression and therapy of cancer. Cell Death Differ 13: 12-19, 2006.

5. Busam KJ, Kucukgol D, Eastlake-Wade S, et al: Clusterin expression in primary and metastatic melanoma. J Cutan Pathol 33: 619-623, 2006.

6. Bi J, Guo AL, Lai YR, et al: Overexpression of clusterin correlates with tumor progression, metastasis in gastric cancer: a study on tissue microarrays. Neoplasma 57: 191-197, 2010.

7. Miyake H, Gleave ME, Arakawa S, et al: Introducing the clusterin gene into human renal cell carcinoma cells enhances their metastatic potential. J Urol 167: 2203-2208, 2002.

8. Wei L, Xue T, Wang J, et al: Roles of clusterin in progression, chemoresistance and metastasis of human ovarian cancer. Int J Cancer 125: 791-806, 2009.

9. Bai J and Hu S: Transcriptome network analysis reveals potential candidate genes for squamous lung cancer. Int J Mol Med 29 95-101, 2012

10. Sakai I, Miyake H, Takenaka A and Fujisawa M: Expression of potential molecular markers in renal cell carcinoma: impact on clinicopathological outcomes in patients undergoing radical nephrectomy. BJU Int 104: 942-946, 2009.
11. Kususda Y, Miyake H, Gleave ME and Fujisawa M: Clusterin inhibition using OGX-011 synergistically enhances antitumour activity of sorafenib in a human renal cell carcinoma model. Br J Cancer 106: 1945-1952, 2012.

12. Hanahan D and Weinberg RA: Hallmarks of cancer: the next generation. Cell 144: 646-674, 2011

13. Shaikhibrahim Z, Lindstrot A, Langer B, Buettner R and Wernert N: Comprehensive gene expression microarray analysis of Ets-1 blockade in PC3 prostate cancer cells and correlations with prostate cancer tissues: Insights into genes involved in the metastatic cascade. Int J Mol Med 27: 811-819, 2011.

14. Tabuchi Y, Wada S, Furusawa Y, Ohtsuka K and Kondo T: Gene networks related to the cell death elicited by hyperthermia in human oral squamous cell carcinoma HSC-3 cells. Int J Mol Med 29: 380-386, 2012.

15. Beroukhim R, Brunet JP, Di Napoli A, et al: Patterns of gene expression and copy-number alterations in von-hippel lindau disease-associated and sporadic clear cell carcinoma of the kidney. Cancer Res 69: 4674-4681, 2009.

16. Monzon FA, Alvarez K, Gatalica Z, et al: Detection of chromosomal aberrations in renal tumors: a comparative study of conventional cytogenetics and virtual karyotyping with single-nucleotide polymorphism microarrays. Arch Pathol Lab Med 133: 1917-1922, 2009. 